\title{
SOME EXACT BIANCHI TYPES COSMOLOGIES IN SCALAR TENSOR THEORY OF GRAVITATION
}

\author{
A. H. HASMANI, AHMED M. AL-HAYSAH* \\ Department of Mathematics, Sardar Patel University, Vallabh Vidyanagar-388120, Gujarat, India \\ *Corresponding Author: alhaysah2017@spuvvn.edu \\ Received Nov. 15, 2018
}

\begin{abstract}
AвSTRACT. In this paper, we attempt to study spatially homogeneous Bianchi types-I, III, $\mathrm{V}, \mathrm{VI}_{0} \& \mathrm{VI}_{h}$ cosmological models in scalar-tensor theory (STT) of gravity. We discuss three types of average scale-factor solutions for some Bianchi types cosmological models using a special law for the deceleration parameter (DP) which is linear in time with a negative slope. The exact solutions of the Einstein's field equations (EFEs) have been obtained for three different physically viable cosmologies. The physical and geometrical properties of the cosmological models have been discussed.
\end{abstract} 2010 Mathematics Subject Classification. 83D05.

Key words and phrases. Bianchi types-I, III, $\mathrm{V}, \mathrm{VI}_{0} \& \mathrm{VI}_{h}$; ST theory of gravity; cosmological models.

\section{INTRODUCTION}

Einstein's general theory of relativity (GR) is a geometrical theory of space-times. The fundamental building block is a metric tensor field $g_{i j}$ which is a tensor of rank two. In an alternative theory based on a metric tensor field along with another dynamical scalar field (as the name suggests) coupled to it is proposed by Brans and Dicke [4] accordingly called STT of gravity. The scalar-tensor theory of gravity had earlier been attempted by G. Nodstrom by promoting the Newtonian potential function to a Lorentz scalar [20].

The theory formulated in [4] which is an alternative theory of gravitation called STT in which the tensor field alone is geometrized and the scalar field is aligned to the geometry. Sen and Dunn [23] have proposed a new STT of gravitation in which both the scalar and tensor fields have intrinsic geometrical significance. The scalar field in this theory is characterized by the function $\phi=\phi\left(x^{i}\right)$ where $x^{i}(\mathrm{i}=1,2,3,4)$ are coordinates in the four-dimensional Lyra manifold and the tensor field is identified with the metric tensor of the manifold.

Nordtvedt (1970) [21] proposed a general class of ST gravitational theories in which the parameter $\omega$ of the Brans and Dicke [4] theory is allowed to be an arbitrary (positive definite) function of the scalar field $(\omega \rightarrow \omega(\phi))$. As was observed by R. Chaubey, and A. Shukla (2013) [5], a new class of Bianchi cosmological models in $f(R, T)$ gravity. The general class of Bianchi cosmological models in Brans-Dicke theory was studied by R. Chaubey, and A. Shukla (2014) [6]

DOI: $10.28924 / \mathrm{APJM} / 6-4$ 
The STT was conceived originally by Jordan frame (JF), who started to embed a four-dimensional curved manifold in five-dimensional flat space-time [15]. He showed that a constraint in formulating projective geometry can be a four-dimensional scalar field, which enables one to describe a space-time dependent gravitational "constant" in accordance with P. A.M Dirac's [7] argument that the gravitational constant should be time-dependent, which is obviously beyond what can be understood within the scope of the standard theory.

In the STT, the scalar field $(\phi)$ plays the role of the (local) gravitational coupling with $G \sim \phi^{-1}$, and consequently the gravitational "constant" is not in fact, a constant but is determined by the total matter in the universe (Mach's principle) through an auxiliary scalar field equation. The scalar field couples to both matter and space-time geometry, the strength of the coupling is represented by an arbitrary dimensionless function $\omega(\phi)$.

In reality, STT is one of the most natural generalizations of the Einstein theory of gravity. Their essence is in one or several scalar fields that are mediators of the gravitational interaction in addition to the space-time metric in the classical Einstein theory. STT can be considered as an Einstein theory of gravity but with variable gravitational constant.

In this paper, an attempt is made to describe some the exact solutions of Bianchi types-I, III, $\mathrm{V}, \mathrm{VI}_{0} \& \mathrm{VI}_{h}$ cosmological models in the framework of STT of gravity. The physical and geometrical properties of such models have also been discussed.

\section{STT of Gravity}

Einstein's celebrated theory of GR currently provides a fairly good understanding of gravity. It describes the curvature of space-times and the motion of objects in such a space-time, as well as how mass and energy influence the curvature itself. GR may be studied in a field theoretic setting, for which the action reads

$$
I_{S T}=S_{E H}+S_{M}\left(g_{i j}, \phi\right)
$$

Here $S_{E H}$ is the Einstein-Hilbert action that describes the dynamics of the metric $g_{i j}$ via the Ricci scalar $R$. The second term, $S_{M}$ is the action and Lagrangian density for matter field field $\phi$. The STT of gravity is a modification of GR, and the action integral in the JF of such gravitational STT can be written as follows

$$
I_{S T}=\frac{1}{16 \pi} \int d^{4} x \sqrt{-g}\left[\phi R-\frac{\omega(\phi)}{\phi} \nabla_{i} \phi \nabla^{j} \phi\right]+S_{M}\left(g_{i j}, \phi\right)
$$

where

$$
S_{M}=\int d^{4} x \sqrt{-g} L_{m}
$$

and $g$ is the determinant of the metric, $\sqrt{-g} d^{4} x$ is the 4-dimensional volume element, $\phi$ is the scalar field, $\omega(\phi)$ is some function of $\phi, \nabla_{i}$ is the covariant derivative operator. The energy-momentum tensor $T_{i j}$ is defined from the Lagrangian density $S_{M}$ as [17]

$$
T_{i j}=\frac{-2}{\sqrt{-g}} \frac{\partial\left(\sqrt{-g} L_{M}\right)}{\partial g^{i j}} .
$$


By varying the action $I_{S T}$ and using the properties

$$
\begin{aligned}
\partial(\sqrt{-g}) & =-\frac{1}{2} \sqrt{-g} g_{i j} \partial g^{i j}, \\
\partial(R \sqrt{-g}) & =\sqrt{-g} G_{i j} \partial g^{i j} .
\end{aligned}
$$

The field Equation (2.2) reduces to

$$
G_{i j}+\frac{\omega(\phi)}{\phi^{2}}\left[\nabla_{i} \phi \nabla_{j} \phi-\frac{1}{2} g_{i j} \nabla_{k} \phi \nabla^{k} \phi\right]+\frac{1}{\phi}\left[\nabla_{i} \nabla_{j} \phi-g_{i j} \square \phi\right]=\frac{8 \pi}{\phi} T_{i j},
$$

where $G_{i j}$ is the Einstein tensor, $\square=\nabla^{k} \nabla_{k}=g^{k l} \nabla_{l} \nabla_{k}$ is the d'Alembert operator for a scalar field. One can take the trace of Equation (2.6) overall space to $g^{i j}$, by using $g^{i j} G_{i j}=-R$, we obtain

$$
(2 \omega(\phi)+3) \square \phi=8 \pi T-\frac{d \omega}{d \phi} \nabla_{k} \phi \nabla^{k} \phi,
$$

where $T=g^{i j} T_{i j}$ is the trace of the stress-energy. Also the following conservation equations are satisfied

$$
\nabla_{i} T^{i j}=0
$$

The conservation equation given above implies that the test-particles describes geodesics as in the case of GR.

\section{Exact Bianchi Types-I, III, V, $\mathrm{VI}_{0} \& \mathrm{VI}_{h}$ Solutions}

Here we first develop some important cosmological parameters and field equations for Bianchi types-I, III, V, $\mathrm{VI}_{0} \& \mathrm{VI}_{h}$ space-time and then find the exact solutions of field equations for constant and non-constant curvature case.

3.1. The Metric and The Field Equations. The spatially homogeneous (SH) and anisotropic Bianchi types space-times are given by,

$$
d s^{2}=d t^{2}-A_{1}^{2} d x^{2}-\mathrm{e}^{-2 \alpha x} A_{2}^{2} d y^{2}-\mathrm{e}^{-2 \beta x} A_{3}^{2} d z^{2},
$$

where $A_{1}, A_{2}$, and $A_{3}$ are called cosmic scale-factors which are functions of time $\mathrm{t}$, and $(x, y, z)$ are the Eulerian angles, so the equation (3.1) represents different Bianchi types as,

\begin{tabular}{|c|c|c|c|c|c|}
\hline Bianchi type & $I$ & $I I I$ & $V$ & $V I_{0}$ & $V I_{h}$ \\
\hline$\alpha$ & 0 & 0 & 1 & 1 & 1 \\
\hline$\beta$ & 0 & 1 & 1 & -1 & $\mathrm{~h}-1$ \\
\hline
\end{tabular}

TABLE 1. Canonical structure constants for some Bianchi types in such a space-time.

The computations of relevant tensors to using Mathematica [12] and [13]; for metric (3.1) gives,

$$
R=2\left[\frac{\ddot{A_{1}}}{A_{1}}+\frac{\ddot{A_{2}}}{A_{2}}+\frac{\ddot{A_{3}}}{A_{3}}+\frac{\dot{A_{1}} \dot{A_{2}}}{A_{1} A_{2}}+\frac{\dot{A_{1}} \dot{A_{3}}}{A_{1} A_{3}}+\frac{\dot{A_{2}} \dot{A_{3}}}{A_{2} A_{3}}-\frac{\alpha^{2}+\beta^{2}+\alpha \beta}{A_{1}^{2}}\right] .
$$


where an overhead dot denotes derivative with respect to time $t$. The energy -momentum tensor for a perfect fluid which will be assumed here, is written in the form

$$
T_{i j}=(\rho+p) u_{i} u_{j}-p g_{i j}
$$

where $\rho$ is the proper energy density, $p$ is the isotropic pressure and $u_{i}=(0,0,0,1)$ is 4 -velocity of the fluid particles which satisfies the condition $u^{i} u_{i}=1$. The average scale-factor $a(t)$ and spatial volume $V$ are defined by,

$$
V=a^{3}=\prod_{i=1}^{3} A_{i}
$$

Mean Hubble parameter(HP) is given by ,

$$
H=\frac{\dot{a}}{a}=\frac{1}{3} \frac{\dot{V}}{V}=\frac{1}{3} \sum_{i=1}^{3} H_{i}=\frac{1}{3}\left(\frac{\dot{A_{1}}}{A_{1}}+\frac{\dot{A_{2}}}{A_{2}}+\frac{\dot{A_{3}}}{A_{3}}\right) .
$$

The directional HPs $H_{i},(i=1,2,3)$ have values given by,

$$
H_{i}=\frac{\dot{A}_{i}}{A_{i}}, i=1,2,3 \text {. }
$$

The cosmological parameters such as the scalar expansion $(\theta)$, shear $\left(\sigma^{2}\right)$, shear parameter $\left(\Sigma^{2}\right)$ are given by,

$$
\begin{aligned}
\theta & =3 H=\left(\frac{\dot{A_{1}}}{A_{1}}+\frac{\dot{A_{2}}}{A_{2}}+\frac{\dot{A_{3}}}{A_{3}}\right), \\
\sigma^{2} & =\frac{1}{2} \sigma_{i j} \sigma^{i j}=\frac{1}{2}\left[\sum_{i=1}^{3} H_{i}^{2}-3 H^{2}\right], \\
\Sigma^{2} & =\Sigma_{+}^{2}+\Sigma_{-}^{2}=\frac{\sigma^{2}}{3 H^{2}}=\frac{1}{6} \Sigma_{i j} \Sigma^{i j}, \text { with } \Sigma_{i j}=\frac{\sigma_{i j}}{H} .
\end{aligned}
$$

The density parameter $\Omega$ is given by,

$$
\Omega=1-\Sigma^{2}-K \geq 0
$$

where the curvature parameter $K$ is given by [24],

$$
K=\frac{{ }^{3} R}{6 H^{2}}=\frac{1}{12}\left(\sum_{i} N_{i}^{2}-2 \sum_{i<j} N_{i} N_{j}\right), i, j=1,2,3,
$$

i.e.,

\begin{tabular}{|c|c|c|c|c|}
\hline Group class & Bianchi type & $N_{1}$ & $N_{2}$ & $N_{3}$ \\
\hline Class A, $(a=0)$ & $I$ & 0 & 0 & 0 \\
\cline { 2 - 5 } & $V I_{0}$ & 0 & + & - \\
\hline \multirow{3}{*}{ class B, $(a \neq 0)$} & $\mathrm{V}$ & 0 & 0 & 0 \\
\cline { 2 - 5 } & $V I_{h}$ & 0 & - & + \\
\cline { 2 - 5 } & $I I I$ & 0 & + & - \\
\hline
\end{tabular}

TABLE 2. Canonical structure constants for different Bianchi types. 
The three structure constants $N_{1}, N_{2}$, and $N_{3}$ are the eigenvalues of the symmetric matrix, $N^{i j}=\operatorname{diag}\left(N_{1}, N_{2}, N_{3}\right)$. Here, we have considered the linearly time-varying DP in the form as given in [1] and [22], also discussion is based on them,

$$
q=-1-\frac{\dot{H}}{H^{2}}=-\frac{\ddot{a} a}{\dot{a}^{2}}=-k t+m-1 .
$$

where $k$ and $m$ are positive constants. We see that the DP is linear in time with a negative slope. The sign of $q$ indicates whether the model accelerates or not, and the positive sign of $q$ corresponds to the standard decelerating model, whereas the negative sign indicates accelerated expansion. For $m>1+k t, q>0$, therefore the model represents a decelerating model, whereas for $k t<m \leq 1+k t$, we get $-1 \leq q<0$, which describes an accelerating model of the universe. Solving Equation (3.12) for the scale-factor $a(t)$, we obtain the law of variation for average scale-factor $a$ as

$$
a= \begin{cases}k_{1} \mathrm{e}^{l t} & \text { for } k=0, m=0, \\ \left(m l t+k_{2}\right)^{\frac{1}{m}} & \text { for } k=0, m>0, \\ k_{3} \mathrm{e}^{\frac{2}{m} \tanh ^{-1}\left(\frac{k t}{m}-1\right)} & \text { for } k>0, m>1 .\end{cases}
$$

where $k_{1}, k_{2}$ and $k_{3}$ are constants of integration. thus we obtain three values of the average scale-factor $a$ that correspond to three different models of the universe. In this paper, we consider the average scale-factor $a$ for all cases.

\section{Exact Solution for Some Bianchi Types}

Here we first develop some important cosmological parameters and EFEs for Bianchi types-I, III, $\mathrm{V}, \mathrm{VI}_{0} \& \mathrm{VI}_{h}$ space-times and then find the exact solutions of EFEs for constant and non-constant curvature cases.

Using Equation (2.6),(3.1) and (3.3), we obtain a set of differential equations for Bianchi types-I, III, V, $\mathrm{VI}_{0} \& \mathrm{VI}_{h}$ space-times,

$$
\begin{aligned}
& \frac{\ddot{A_{2}}}{A_{2}}+\frac{\ddot{A_{3}}}{A_{3}}+\frac{\dot{A_{2}} \dot{A_{3}}}{A_{2} A_{3}}-\frac{\alpha \beta}{A_{1}^{2}}+\frac{\omega}{2}\left(\frac{\dot{\phi}}{\phi}\right)^{2}+\frac{\ddot{\phi}}{\phi}+\left(\frac{\dot{A_{2}}}{A_{2}}+\frac{\dot{A_{3}}}{A_{3}}\right) \frac{\dot{\phi}}{\phi}=-\frac{8 \pi p}{\phi}, \\
& \frac{\ddot{A_{1}}}{A_{1}}+\frac{\ddot{A_{3}}}{A_{3}}+\frac{\dot{A_{1}} \dot{A_{3}}}{A_{1} A_{3}}-\frac{\beta^{2}}{A_{1}^{2}}+\frac{\omega}{2}\left(\frac{\dot{\phi}}{\phi}\right)^{2}+\frac{\ddot{\phi}}{\phi}+\left(\frac{\dot{A_{1}}}{A_{1}}+\frac{\dot{A_{3}}}{A_{3}}\right) \frac{\dot{\phi}}{\phi}=-\frac{8 \pi p}{\phi}, \\
& \frac{\ddot{A_{1}}}{A_{1}}+\frac{\ddot{A_{2}}}{A_{2}}+\frac{\dot{A_{1}} \dot{A_{2}}}{A_{1} A_{2}}-\frac{\alpha^{2}}{A_{1}^{2}}+\frac{\omega}{2}\left(\frac{\dot{\phi}}{\phi}\right)^{2}+\frac{\ddot{\phi}}{\phi}+\left(\frac{\dot{A_{1}}}{A_{1}}+\frac{\dot{A_{2}}}{A_{2}}\right) \frac{\dot{\phi}}{\phi}=-\frac{8 \pi p}{\phi}, \\
& \frac{\dot{A_{1}} \dot{A_{2}}}{A_{1} A_{2}}+\frac{\dot{A_{1}} \dot{A_{3}}}{A_{1} A_{3}}+\frac{\dot{A_{2}} \dot{A_{3}}}{A_{2} A_{3}}-\frac{\alpha^{2}+\beta^{2}+\alpha \beta}{A_{1}^{2}}-\frac{\omega}{2}\left(\frac{\dot{\phi}}{\phi}\right)^{2}+\left(\frac{\dot{A_{1}}}{A_{1}}+\frac{\dot{A_{2}}}{A_{2}}+\frac{\dot{A_{3}}}{A_{3}}\right) \frac{\dot{\phi}}{\phi}=\frac{8 \pi \rho}{\phi}, \\
& (\alpha+\beta) \frac{\dot{A_{1}}}{A_{1}}-\alpha \frac{\dot{A_{2}}}{A_{2}}-\beta \frac{\dot{A_{3}}}{A_{3}}=0 .
\end{aligned}
$$

and

$$
\frac{\ddot{\phi}}{\phi}+\left(\frac{\dot{A_{1}}}{A_{1}}+\frac{\dot{A_{2}}}{A_{2}}+\frac{\dot{A_{3}}}{A_{3}}\right) \frac{\dot{\phi}}{\phi}=\frac{8 \pi(\rho-3 p)}{(3+2 \omega) \phi} .
$$


Now using Equations (4.1) to (4.4), we obtain the continuity equation as follows

$$
\dot{\rho}+(\rho+p)\left(\frac{\dot{A_{1}}}{A_{1}}+\frac{\dot{A_{2}}}{A_{2}}+\frac{\dot{A_{3}}}{A_{3}}\right)+\left(\frac{\dot{A_{1}}}{A_{1}}+\frac{\dot{A_{2}}}{A_{2}}+\frac{\dot{A_{3}}}{A_{3}}\right)=0 .
$$

Now, by adding three times Equation (4.4) and Equations (4.1), (4.2) and (4.3), one can easily obtain

$$
\begin{aligned}
\frac{\ddot{A_{1}}}{A_{1}}+\frac{\ddot{A_{2}}}{A_{2}}+\frac{\ddot{A_{3}}}{A_{3}} & +2\left(\frac{\dot{A_{1}} \dot{A_{2}}}{A_{1} A_{2}}+\frac{\dot{A_{1}} \dot{A_{3}}}{A_{1} A_{3}}+\frac{\dot{A_{2}} \dot{A_{3}}}{A_{2} A_{3}}-\frac{\alpha^{2}+\beta^{2}+\alpha \beta}{A_{1}^{2}}\right) \\
& +\frac{3 \ddot{\phi}}{2 \phi}+\frac{5}{2}\left(\frac{\dot{A_{1}}}{A_{1}}+\frac{\dot{A_{2}}}{A_{2}}+\frac{\dot{A_{3}}}{A_{3}}\right) \frac{\dot{\phi}}{\phi}=-\frac{12 \pi(\rho-p)}{\phi} .
\end{aligned}
$$

From Equation (3.5), we find

$$
\frac{\ddot{V}}{V}=\frac{\ddot{A_{1}}}{A_{1}}+\frac{\ddot{A_{2}}}{A_{2}}+\frac{\ddot{A_{3}}}{A_{3}}+2\left(\frac{\dot{A_{1}} \dot{A_{2}}}{A_{1} A_{2}}+\frac{\dot{A_{1}} \dot{A_{3}}}{A_{1} A_{3}}+\frac{\dot{A_{2}} \dot{A_{3}}}{A_{2} A_{3}}\right) .
$$

From Equations (4.9) and (4.8), one can easily obtain

$$
\frac{\ddot{V}}{V}-\frac{2\left(\alpha^{2}+\beta^{2}+\alpha \beta\right)}{A_{1}^{2}}+\frac{3 \ddot{\phi}}{2 \phi}+\frac{5}{2}\left(\frac{\dot{V}}{V}\right) \frac{\dot{\phi}}{\phi}=-\frac{12 \pi(\rho-p)}{\phi} .
$$

Integrating Equation (4.5) and absorbing the integrating constant into $A_{2}$ or $A_{3}$, we get,

$$
A_{1}^{\alpha+\beta}=A_{2}^{\alpha} A_{3}^{\beta} \text {. }
$$

The expression of $V$ given in (3.4) when used in (4.11) gives

$$
A_{1}^{\beta+2 \alpha}=a^{3 \alpha} A_{3}^{\beta-\alpha} .
$$

Setting $A_{3}=V^{d}$, where $d$ is any constant, then from Equation (4.12), we get

$$
A_{1}^{\beta+2 \alpha}=a^{3 \alpha} V^{d(\beta-\alpha)}=a^{3(\alpha+\beta d-\alpha d)} .
$$

4.1. Cosmological Solutions for $k=0, m=0$. We now obtain physically factual cosmological models to describe the decelerating and accelerating phases of the universe. The necessary computations were done using Mathematica, and also it was used to plot graphs. Using Equations (3.4), (3.13)and (4.13), the metric coefficients $A_{i}(i=1,2,3)$ turn out to be

$$
A_{i}(t)=\left(k_{1} \mathrm{e}^{l t}\right)^{\xi_{i}}, i=1,2,3,
$$

where

$$
\xi_{1}=\frac{3(\alpha+\beta d-\alpha d)}{\beta+2 \alpha}, \xi_{2}=\frac{3(\alpha-2 \beta d-\alpha d+\beta)}{\beta+2 \alpha}, \xi_{3}=3 d,
$$

and $\xi_{1}+\xi_{2}+\xi_{3}=3$. Using these in (3.1), we get the following form of the metric (3.1),

$$
d s^{2}=d t^{2}-\left(k_{1} \mathrm{e}^{l t}\right)^{2 \xi_{1}} d x^{2}-\mathrm{e}^{-2 \alpha x}\left(\left(k_{1} \mathrm{e}^{l t}\right)^{2 \xi_{2}} d y^{2}-\mathrm{e}^{-2 \beta x}\left(k_{1} \mathrm{e}^{l t}\right)^{2 \xi_{3}} d z^{2} .\right.
$$

The spatial volume and the average scale-factor $a(t)$ are given by

$$
V=\left(k_{1} \mathrm{e}^{l t}\right)^{3}=a^{3} .
$$


Mean HP and DP take the form

$$
H=l, q=\frac{-\ddot{a}}{a H^{2}}=-1 .
$$

The universe would exhibit de-Sitter expansion if $q=-1$ (also known as exponential expansion), the case corresponds to the law of Berman's [2,3] of constant DP. Using this law one can generalize the cosmological solutions that are obtained via constant DP. Furthermore, one can obtain models that fit better the cosmological data. The HPs in the directional of $x, y$ and $z$ axes are

$$
H_{i}=\frac{\dot{A}_{i}}{A_{i}}=l \xi_{i}, i=1,2,3
$$

The scalar expansion is

$$
\theta=3 l=3 H .
$$

The shear scalar $\left(\sigma^{2}\right)$, shear parameter $\left(\Sigma^{2}\right)$ and density parameter $(\Omega)$ are

$$
\begin{aligned}
\sigma^{2} & =\frac{l^{2}}{2}\left[\xi_{1}^{2}+\xi_{2}^{2}+\xi_{3}^{2}-3\right], \\
\Sigma^{2} & =\frac{1}{6}\left[\xi_{1}^{2}+\xi_{2}^{2}+\xi_{3}^{2}-3\right], \\
\Omega & =1-\frac{1}{6}\left[\xi_{1}^{2}+\xi_{2}^{2}+\xi_{3}^{2}-3\right]-K \geq 0,
\end{aligned}
$$

where $K$ is the curvature parameter, as defined in Equation (3.11).

The Ricci scalar $R$ for Bianchi types-I, III, $\mathrm{V}, \mathrm{VI}_{0} \& \mathrm{VI}_{h}$ cosmological models is given by Equations (4.14) and (3.2), it follows that

$$
R=2\left[k_{1}^{-2 \xi_{1}} \mathrm{e}^{-2 l t \xi_{1}}\left(\alpha^{2}+\beta^{2}+\alpha \beta\right)+l^{2}\left(\xi_{1}^{2}+\xi_{2}^{2}+\xi_{3}^{2}+\xi_{1} \xi_{2}+\xi_{1} \xi_{3}+\xi_{2} \xi_{3}\right)\right] .
$$

The energy density $\rho$ in the model is obtained as

$$
\rho=\frac{\phi}{8 \pi}\left[k_{1}^{-2 \xi_{1}} \mathrm{e}^{-2 l t \xi_{1}}\left(\alpha^{2}+\beta^{2}+\alpha \beta\right)+l^{2}\left(\xi_{1} \xi_{2}+\xi_{1} \xi_{3}+\xi_{2} \xi_{3}\right)+\frac{3 l \dot{\phi}}{\phi}-\frac{\omega}{2}\left(\frac{\dot{\phi}}{\phi}\right)^{2}\right] .
$$

The expressions for isotropic pressure $p$ in the model is given by

$$
p=\frac{-\phi}{8 \pi}\left[-k_{1}^{-2 \xi_{1}} \mathrm{e}^{-2 l t \xi_{1}}(\alpha \beta)+l^{2}\left(\xi_{2}^{2}+\xi_{3}^{2}+\xi_{2} \xi_{3}\right)+\frac{l\left(\xi_{2}+\xi_{3}\right) \dot{\phi}}{\phi}+\frac{\omega}{2}\left(\frac{\dot{\phi}}{\phi}\right)^{2}+\frac{\ddot{\phi}}{\phi}\right] .
$$

4.2. Cosmological Solutions for $k=0, m>0$. We now obtain physically factual cosmological models to describe the decelerating and accelerating phases of the universe. Using Equations (3.4), (3.13) and (4.13), the metric coefficients $A_{i}(i=1,2,3)$ turn out to be

$$
A_{i}(t)=\left(m l t+k_{2}\right)^{\frac{\xi_{i}}{m}}, i=1,2,3
$$

where $\xi_{i}, i=1,2,3$ are similar to those written earlier in Equation (4.15). Using these in (3.1), we get the following form of the metric (3.1),

$$
d s^{2}=d t^{2}-\left(m l t+k_{2}\right)^{\frac{2 \xi_{1}}{m}} d x^{2}-\mathrm{e}^{-2 \alpha x}\left(m l t+k_{2}\right)^{\frac{2 \xi_{2}}{m}} d y^{2}-\mathrm{e}^{-2 \beta x}\left(m l t+k_{2}\right)^{\frac{2 \xi_{3}}{m}} d z^{2} .
$$


The spatial volume and the average scale-factor $a(t)$ are given by

$$
V=\left(m l t+k_{2}\right)^{\frac{3}{m}}=a^{3} .
$$

Mean HP and DP take the form

$$
H=\frac{l}{\left(m l t+k_{2}\right)}, q=\frac{-\ddot{a}}{a H^{2}}=m-1 .
$$

The universe would exhibit accelerating expansion if $-1<q<0$ ( also know power-law expansion ), the case corresponds to the law of Berman's [2,3] of constant DP. Using this law one can generalize the cosmological solutions that are obtained via constant DP. Furthermore, one can obtain models that fit better the cosmological data. The HPs in the directional of $x, y$ and $z$ axes are

$$
H_{i}=\frac{\dot{A}_{i}}{A_{i}}=\frac{l \xi_{i}}{\left(m l t+k_{2}\right)}, i=1,2,3(\text { no sum }) .
$$

The scalar expansion is

$$
\theta=\frac{3 l}{\left(m l t+k_{2}\right)}=3 H
$$

The shear scalar is

$$
\sigma^{2}=\frac{l^{2}}{2}\left[\frac{\xi_{1}^{2}+\xi_{2}^{2}+\xi_{3}^{2}-3}{\left(m l t+k_{2}\right)^{2}}\right]
$$

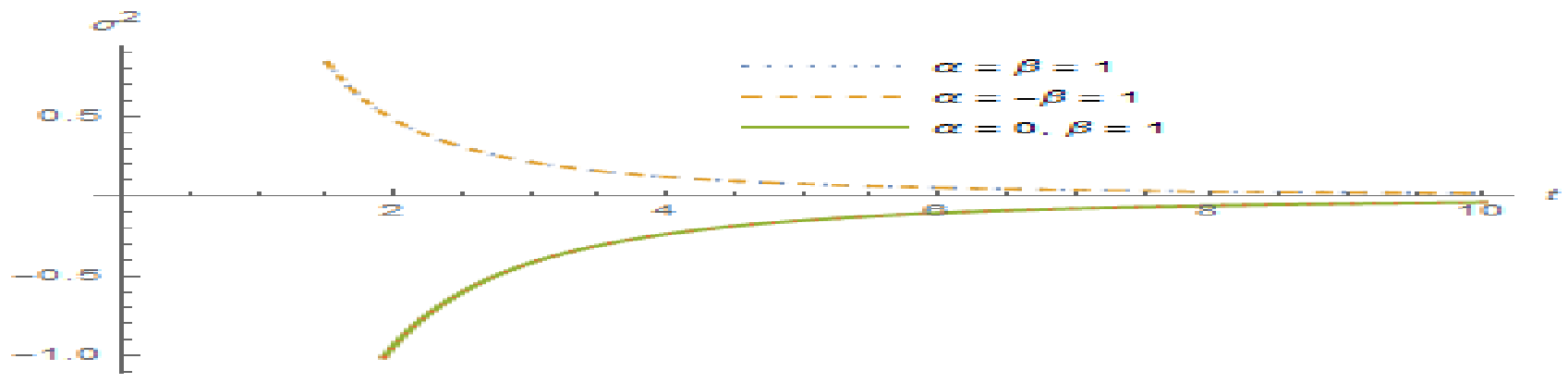

Figure 1. The evolution of shear scalar $\sigma^{2}$ with cosmic time $t$.

Figure 1 shows the evolution of shear scalar $\sigma^{2}$ for some suitable values of constants. From this figure, we observe that the shear scalar is decreasing function of time $t$, i.e. $\sigma^{2} \rightarrow 0$ when $t \rightarrow \infty$. The shear parameter is given by

$$
\Sigma^{2}=\frac{1}{6}\left[\xi_{1}^{2}+\xi_{2}^{2}+\xi_{3}^{2}-3\right]
$$

The density parameter $\Omega$ is given by

$$
\Omega=1-\frac{1}{6}\left[\xi_{1}^{2}+\xi_{2}^{2}+\xi_{3}^{2}-3\right]-K \geq 0
$$


where $K$ is the curvature parameter, as defined in Equation (3.11).

The Ricci scalar $R$ for Bianchi types-I, III, $\mathrm{V}, \mathrm{VI}_{0} \& \mathrm{VI}_{h}$ cosmological models is given by Equations (4.27) and (3.2), it follows that

$$
R=2\left[-\left(m l t+k_{2}\right)^{\frac{-2 \xi_{1}}{m}}\left(\alpha^{2}+\beta^{2}+\alpha \beta\right)+\frac{l^{2}\left(\xi_{1}^{2}+\xi_{2}^{2}+\xi_{3}^{2}+\xi_{1} \xi_{2}+\xi_{1} \xi_{3}+\xi_{2} \xi_{3}-3 m\right)}{\left(m l t+k_{2}\right)^{2}}\right] .
$$

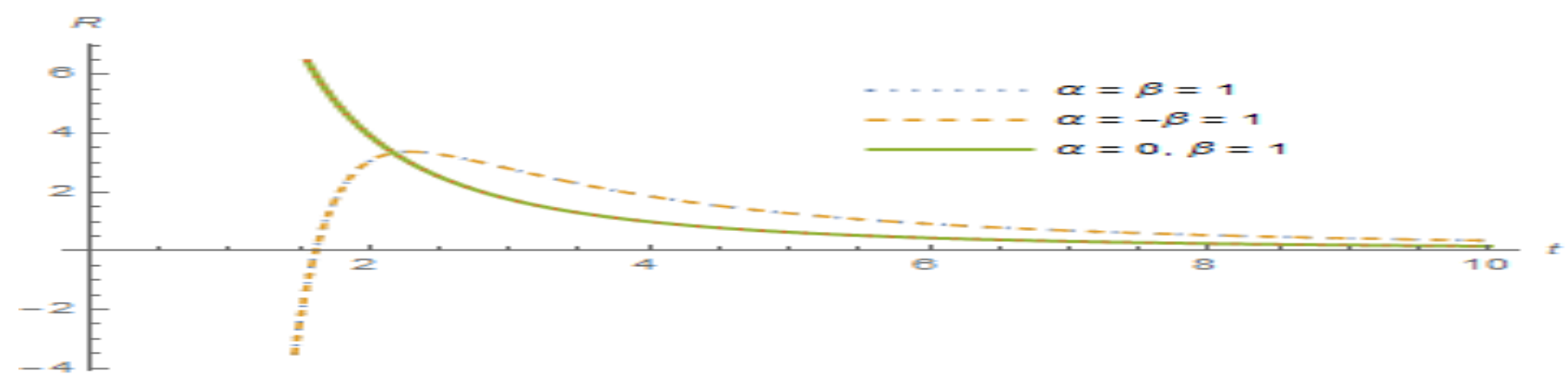

Figure 2. The evolution of Ricci scalar $R$ with cosmic time $t$.

Figure 2 shows the evolution of Ricci scalar $R$ for some suitable values of constants. From this figure, we observe that the Ricci scalar is decreasing function of time $t$, i.e. $R \rightarrow 0$ when $t \rightarrow \infty$. The energy density $\rho$ in the model is obtained as

$$
\begin{aligned}
\rho & =\frac{\phi}{8 \pi}\left[-\left(m l t+k_{2}\right)^{\frac{-2 \xi_{1}}{m}}\left(\alpha^{2}+\beta^{2}+\alpha \beta\right)+\frac{l^{2}\left(\xi_{1} \xi_{2}+\xi_{1} \xi_{3}+\xi_{2} \xi_{3}\right)}{\left(m l t+k_{2}\right)^{2}}\right. \\
& \left.+\frac{3 l}{\left(m l t+k_{2}\right)}\left(\frac{\dot{\phi}}{\phi}\right)-\frac{\omega}{2}\left(\frac{\dot{\phi}}{\phi}\right)^{2}\right] .
\end{aligned}
$$

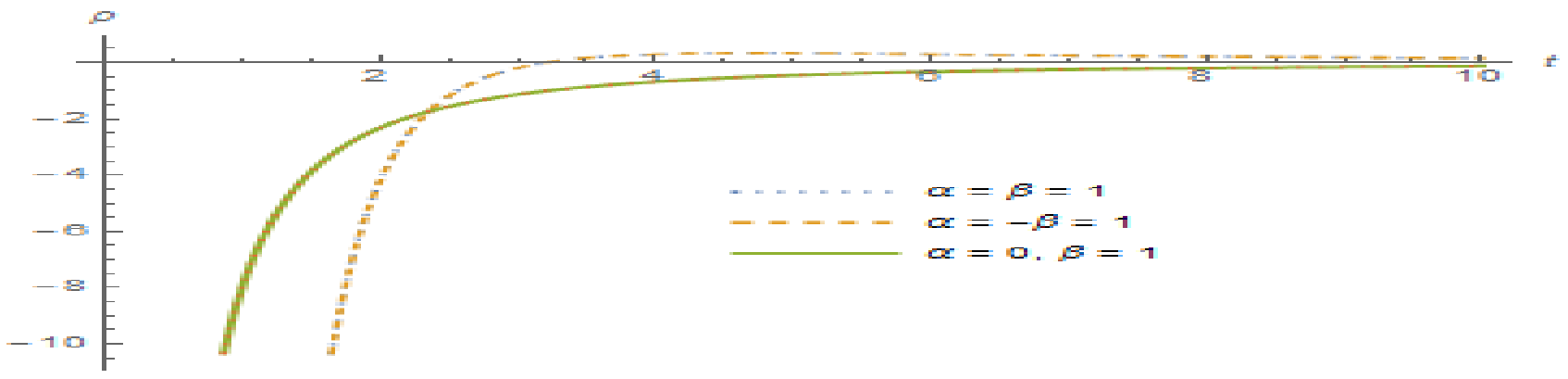

FIGURE 3. The evolution of energy density $\rho$ with cosmic time t.

Figure 3 shows the evolution of energy density $\rho$ for some suitable values of constants. From this figure, we observe that the energy density is decreasing function of time $t$, i.e. $\rho \rightarrow 0$ when $t \rightarrow \infty$. The expressions for 
isotropic pressure $p$ in the model is given by

$$
\begin{aligned}
p & =\frac{-\phi}{8 \pi}\left[-\left(m l t+k_{2}\right)^{\frac{-2 \xi_{1}}{m}}(\alpha \beta)+l^{2}\left(\xi_{2}^{2}+\xi_{3}^{2}+\xi_{2} \xi_{3}-m\left(\xi_{2}+\xi_{3}\right)\right)\right. \\
& \left.+\frac{l\left(\xi_{2}+\xi_{3}\right)}{\left(m l t+k_{2}\right)}\left(\frac{\dot{\phi}}{\phi}\right)+\frac{\omega}{2}\left(\frac{\dot{\phi}}{\phi}\right)^{2}+\frac{\ddot{\phi}}{\phi}\right] .
\end{aligned}
$$

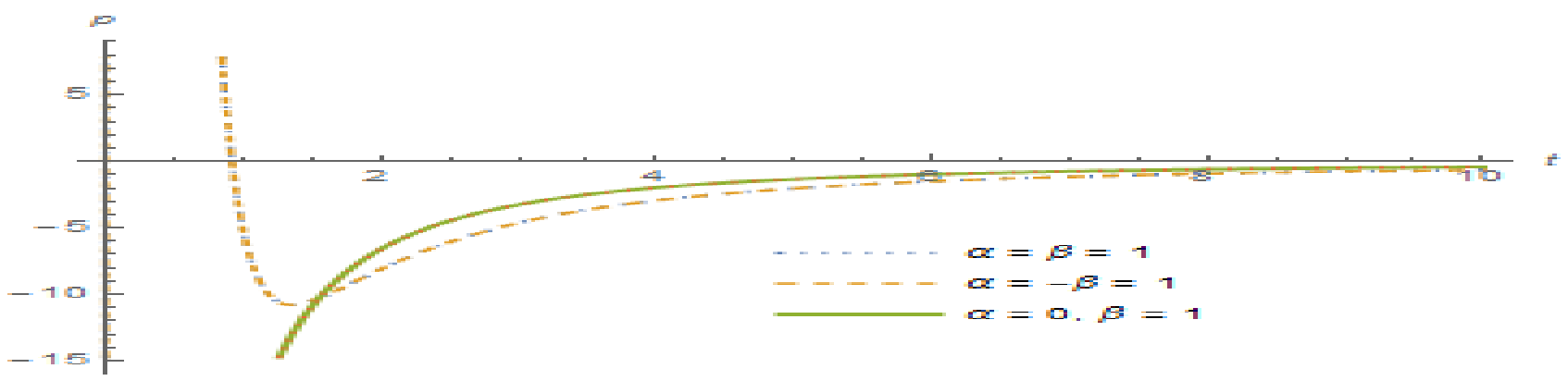

FIGURE 4. The evolution of isotropic pressure $p$ with cosmic time $t$.

Figure 4 shows the evolution of isotropic pressure $p$ for some suitable values of constants. From this figure, we observe that the isotropic pressure is decreasing function of time $t$, i.e. $p \rightarrow 0$ when $t \rightarrow \infty$.

4.3. Cosmological Solutions for $k>0, m>1$. We now obtain physically significant cosmological models to describe the decelerating and accelerating phases of the universe. Using Equations (3.4), (3.13) and (4.13), the metric coefficients $A_{i}(i=1,2,3)$ turn out to be

$$
A_{i}(t)=\left(k_{3} \mathrm{e}^{\frac{2}{m} t a n h^{-1}\left(\frac{k t}{m}-1\right)}\right)^{\xi_{i}}, i=1,2,3
$$

where $\xi_{i}, i=1,2,3$ are similar to those written earlier in Equation (4.15). Using these in (3.1), we get the following form of the metric (3.1),

$$
\begin{aligned}
d s^{2}=d t^{2}-\left(k_{3} \mathrm{e}^{\frac{2}{m} \tanh ^{-1}\left(\frac{k t}{m}-1\right)}\right)^{2 \xi_{1}} d x^{2} & -\mathrm{e}^{-2 \alpha x}\left(\left(k_{3} \mathrm{e}^{\frac{2}{m} \tanh ^{-1}\left(\frac{k t}{m}-1\right)}\right)^{2 \xi_{2}} d y^{2}\right. \\
& -\mathrm{e}^{-2 \beta x}\left(k_{3} \mathrm{e}^{\frac{2}{m} \tanh ^{-1}\left(\frac{k t}{m}-1\right)}\right)^{2 \xi_{3}} d z^{2}
\end{aligned}
$$

The spatial volume and the average scale-factor $a(t)$ are,

$$
V=\left(k_{3} \mathrm{e}^{\frac{2}{m} \tanh ^{-1}\left(\frac{k t}{m}-1\right)}\right)^{3}=a^{3} .
$$




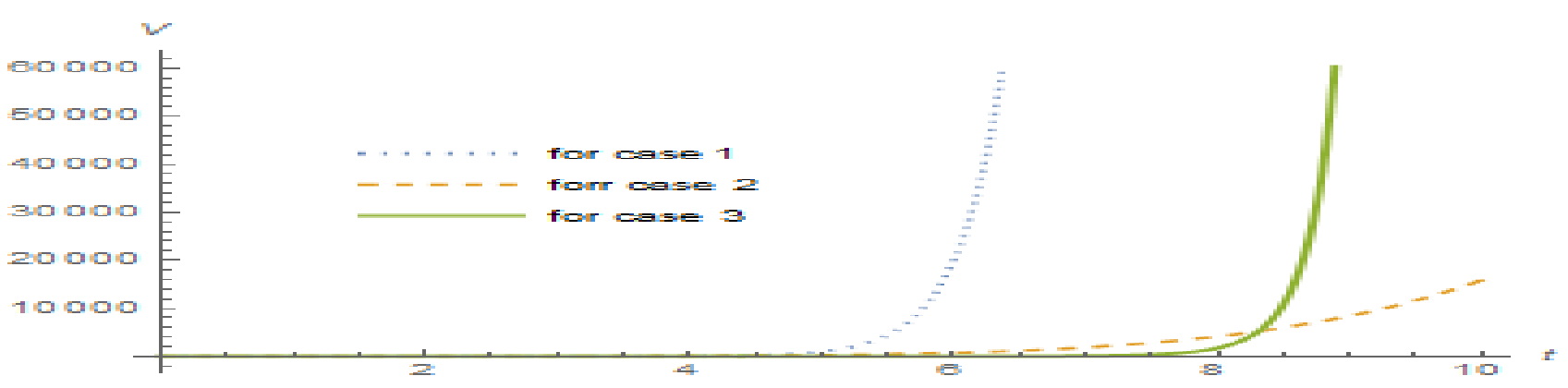

FIGURE 5. The evolution of volume $V$ with cosmic time $t$ in all cases.

Figure 5 shows the evolution of the universe described by Bianchi types-I, III, V, VI $\mathrm{V}_{0} \& \mathrm{VI}_{h}$ models for the DP which is linear in time with negative slope. Mean HP and DP are

$$
H=\frac{2}{t(2 m-k t)}, q=\frac{-\ddot{a}}{a H^{2}}=-k t+m-1 .
$$

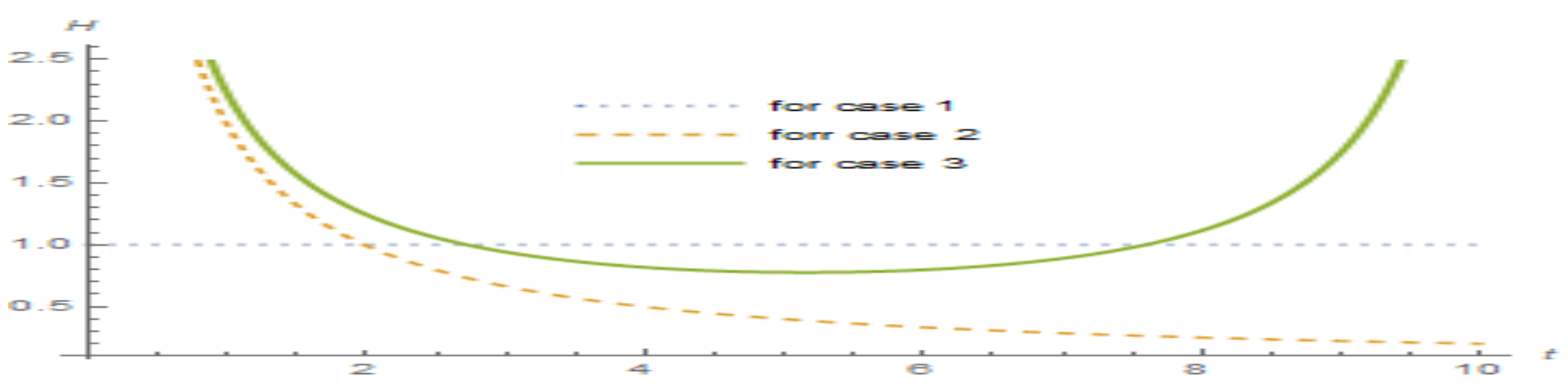

FIGURE 6. The evolution of Mean HP $H$ with cosmic time $t$.

Figure 6 shows the evolution of the HP for some suitable values of constants. From this figure, we observe that the HP is constat and decreasing function of time $t$, i.e. $H \rightarrow 0$ when $t \rightarrow \infty$.

The DP is a linear relationship, $\mathrm{q}$ is greater than zero for some time and then it becomes negative. This the universe star expanding after some time. The elongation is possible due to an increased value of $\mathrm{m}$. The HPs in the directional of $x, y$ and $z$ axes are

$$
H_{i}=\frac{\dot{A}_{i}}{A_{i}}=\frac{2 \xi_{i}}{t(2 m-k t)}, i=1,2,3 .
$$

The scalar expansion is

$$
\theta=\frac{6}{t(2 m-k t)}=3 H .
$$

The shear scalar is

$$
\sigma^{2}=2\left[\frac{\xi_{1}^{2}+\xi_{2}^{2}+\xi_{3}^{2}-3}{t^{2}(k t-2 m)^{2}}\right] .
$$




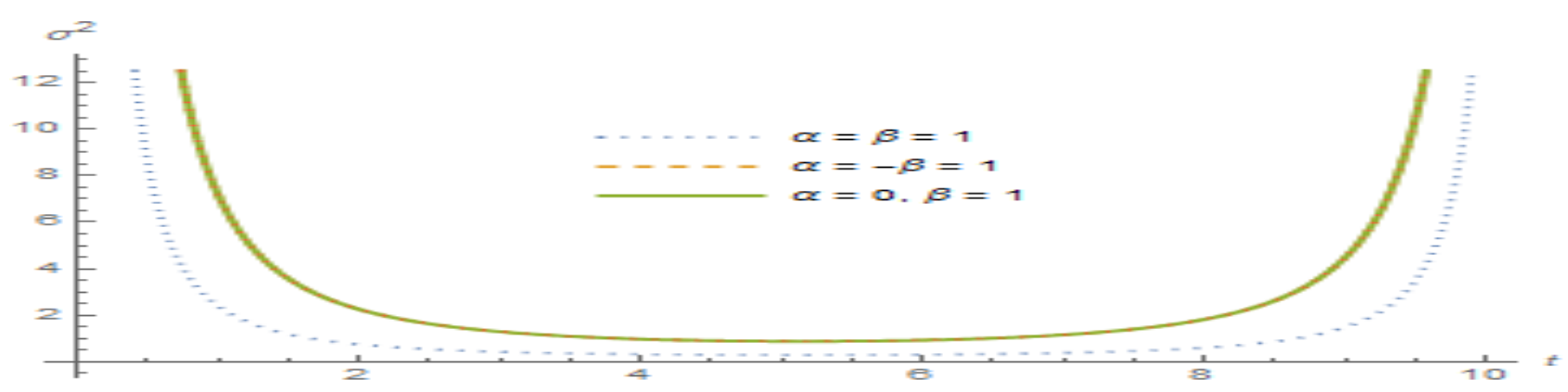

FIgURE 7. The evolution of shear scalar $\sigma^{2}$ with cosmic time $t$.

Figure 7 shows the evolution of shear scalar $\sigma^{2}$ for some suitable values of constants. From this figure, we observe that the shear scalar is decreasing function of time $t$. The shear parameter is given by

$$
\Sigma^{2}=\frac{1}{6}\left[\xi_{1}^{2}+\xi_{2}^{2}+\xi_{3}^{2}-3\right]
$$

The density parameter $\Omega$ is given by

$$
\Omega=1-\frac{1}{6}\left[\xi_{1}^{2}+\xi_{2}^{2}+\xi_{3}^{2}-3\right]-K \geq 0
$$

where $K$ is the curvature parameter, as defined in Equation (3.11).

The Ricci scalar $R$ for Bianchi types-I, III, $\mathrm{V}, \mathrm{VI}_{0} \& \mathrm{VI}_{h}$ cosmological models is given by Equations (4.39) and (3.2), it follows that

$$
\begin{aligned}
R & =2\left[-\left(k_{3} \mathrm{e}^{\frac{2}{m} \tanh ^{-1}\left(\frac{k t}{m}-1\right)}\right)^{-2 \xi_{1}}\left(\alpha^{2}+\beta^{2}+\alpha \beta\right)\right. \\
& \left.+\frac{8\left(\xi_{1}^{2}+\xi_{2}^{2}+\xi_{3}^{2}+\xi_{1} \xi_{2}+\xi_{1} \xi_{3}+\xi_{2} \xi_{3}\right)+3(k t-m)}{t^{2}(k t-2 m)^{2}}\right] .
\end{aligned}
$$

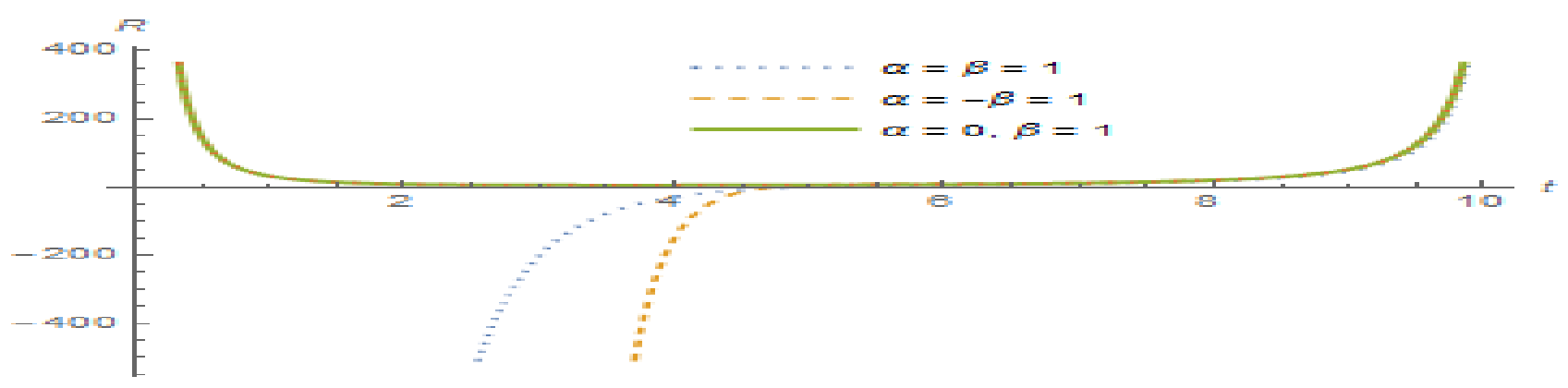

Figure 8. The evolution of Ricci scalar $R$ with cosmic time $t$.

Figure 8 shows the evolution of Ricci scalar $R$ for some suitable values of constants. From this figure, we observe that the Ricci scalar is an increasing and decreasing functions of time $t$ in phases. The energy density $\rho$ 
in the model is obtained as

$$
\begin{aligned}
\rho & =\frac{\phi}{8 \pi}\left[-\left(k_{3} \mathrm{e}^{\frac{2}{m} \tanh ^{-1}\left(\frac{k t}{m}-1\right)}\right)^{-2 \xi_{1}}\left(\alpha^{2}+\beta^{2}+\alpha \beta\right)+\frac{4\left(\xi_{1} \xi_{2}+\xi_{1} \xi_{3}+\xi_{2} \xi_{3}\right)}{t^{2}(k t-2 m)^{2}}\right. \\
& \left.-\frac{6}{t(2 m-k t)}\left(\frac{\dot{\phi}}{\phi}\right)-\frac{\omega}{2}\left(\frac{\dot{\phi}}{\phi}\right)^{2}\right] .
\end{aligned}
$$

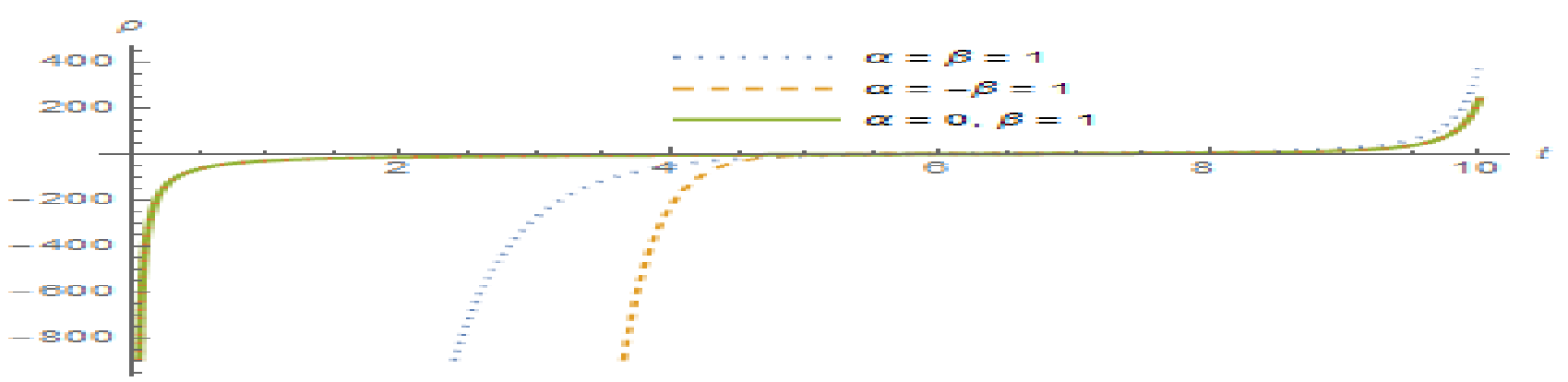

Figure 9. The evolution of energy density $\rho$ with cosmic time t.

Figure 9 shows the evolution of energy density $\rho$ for some suitable values of constants. From this figure, we observe that the energy density is increasing function of time $t$. The expressions for isotropic pressure $p$ in the model is given by

$$
\begin{aligned}
p & =\frac{-\phi}{8 \pi}\left[\frac { 1 } { ( k t - 2 m ) ^ { 2 } } \left(\frac{8\left(\xi_{2}^{2}+\xi_{3}^{2}\right)}{t^{2}}+\frac{8 \xi_{2}\left(k t-m+\xi_{3}\right)}{t^{2}}+\frac{8 \xi_{3}(m-k t)}{t^{2}}\right.\right. \\
& \left.\left.+(k t-2 m)^{2}\left(-2 \alpha \beta\left(k_{3} \mathrm{e}^{\frac{2}{m} \tanh ^{-1}\left(\frac{k t}{m}-1\right)}\right)^{-2 \xi_{1}}+\omega\left(\frac{\dot{\phi}}{\phi}\right)^{2}+2 \frac{\ddot{\phi}}{\phi}\right)\right)\right] .
\end{aligned}
$$

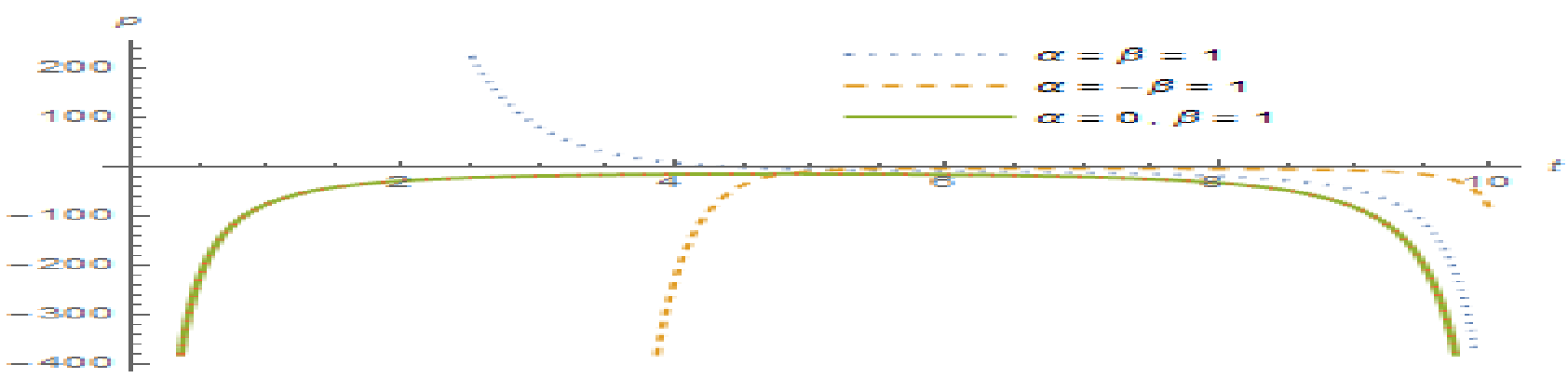

FIGURE 10. The evolution of isotropic pressure $p$ with cosmic time $t$.

Figure 10 shows the evolution of isotropic pressure $p$ for some suitable values of constants. From this figure, we observe that the isotropic pressure is an increasing and decreasing functions of time $t$. 


\section{Discussion And Conclusion}

In this paper, we have extended the study of an exact cosmological solutions of EFEs for Bianchi types-I, III, V , $\mathrm{VI}_{0} \& \mathrm{VI}_{h}$ space-times in STT and obtained solutions for different values of $\alpha$ and $\beta$.

We have also examined the well-known physical and geometrical properties of our models in three different viable cosmologies. It is shown that our models represent expanding, shearing, non-rotating and accelerating universe in each case. The behavior of the cosmological models by analyzing the different physical parameters is in progress.

In the first case, when $k=0$ and $m=0$, for large cosmic time $\mathrm{t}$, the shear scalar tends to zero. Here, $\lim _{t \rightarrow \infty} \frac{\sigma^{2}}{\theta}=0$, so the model approaches isotropy for large cosmic time $t$.

In the second case, when $k=0$ and $m>0$, we observe that the spatial volume $V$ is zero at $t=t_{0}=\frac{-k_{2}}{m l}$. The scalar curvature $R$, the energy density and pressure are infinite at this epoch (Figs. 2, 3, 4). The generalized $\mathrm{HP}$ and directional HPs $H_{i}, i=1,2,3$ are zero as $t \rightarrow \infty$. The rate of expansion is infinite as $t \rightarrow t_{0}$. Thus the universe starts evolving with zero volume at $t=t_{0}=0$, which implies that $k_{2}=0$ and it expands with cosmic time $t$. For large cosmic time $t$, the spatial volume, expansion parameter and shear scalar tend to zero. Here, $\lim _{t \rightarrow \infty} \frac{\sigma^{2}}{\theta}=0$, so the model approaches isotropy for large cosmic time $t$. In other words, the universe evolves from a big bang like situation at $t=t_{0}$ to an isotropic state a fairly large time. Furher at this large time expansion stops.

In the third case, when $k>0$ and $m>1$, for large cosmic time t, the shear scalar tends to zero (Fig. 7). Here, $\lim _{t \rightarrow \infty} \frac{\sigma^{2}}{\theta}=0$, so the model approaches isotropy for large cosmic time $t$.

In each case, the conditions of homogeneity and isotropization, formulated by Collins \& Hawking (1973), are satisfied in the present models. As well, the spatial volume, expansion parameter and shear scalar tended to zero for large cosmic time, $t$. All the physical parameters were calculated and discussed for each model, and in each case, the cosmological model approach isotropy for a large value of cosmic time $t$. The model had a point singularity, and the rate of expansion slowed down and vanished as $t \rightarrow \infty$. This model represents a shearing, non-rotating and expanding universe, which approaches isotropy for large values of $t$. This means that in all cases the evolution of the universe is forwards isotropization. [In other words though at present the universe seems to be anisotropic in a long run it will be isotropic]

\section{Acknowledgments}

AMA is thankful to the Al-Bayda University, and Government of the Republic of Yemen for providing financial support. The authors are thankful to the University Grant Commission, India for providing financial support under UGC-SAP-DRS(III) provided to the Department of Mathematics, Sardar Patel University, Vallabh Vidyanagar, where the work was carried out.

\section{REFERENCES}

[1] O. Akarsu and T. Dereli, Cosmological models with linearly varying deceleration parameter, Int. J. Theor. Phys. 51 (2012), $612-621$.

[2] M. S. Berman, A special law of variation for Hubble's parameter, Il Nuovo Cimento B (1971-1996), 74 (1983), 182-186.

[3] M. S. Berman and F. de Mello Gomide, Cosmological models with constant deceleration parameter, Gen. Relativ. Gravitation 20 (1988), 191-198.

[4] C. Brans and R. H. Dicke, Mach's principle and a relativistic theory of gravitation, Phys. Rev. 124 (1961), 925-935. 
[5] Chaubey, R., and Shukla, A. A new class of Bianchi cosmological models in $f(R, T)$ gravity. Astrophys. Space Sci. 343, 1 (2013), 415-422.

[6] R. Chaubey and A. K. Shukla, The general class of Bianchi cosmological models with viscous fluid and particle creation in Brans-Dicke theory, Res. Astron. Astrophys. 14 (2014), 533-552.

[7] P. A. Dirac, A new basis for cosmology, Proc. Royal Soc. London. Ser. A, Math. Phys. Sci. (1938), 199-208.

[8] G. F. Ellis, Relativistic cosmology, Cargese lect. phys. 6 (1973), 1-60.

[9] V. Faraoni, The $\omega(\phi)$ limit of Brans-Dicke theory, Phys. Lett. A, 245 (1998), 26-30.

[10] . Gron and S. Hervik, Einstein's general theory of relativity: with modern applications in cosmology, Springer Science \& Business Media, 2007.

[11] T. Harko and F. S. Lobo, Generalized dark gravity, Int. J. Modern Phys. D, 21 (2012),1242019.

[12] A. Hasmani, Algebraic computation of newmann-penrose scalars in general relativity using mathematica, J. Sci. 1 (2010), $82-83$.

[13] A. Hasmani and G. Rathva, Algebric computations in general relativity using mathemetica, Prajna J. Pure Appl. Sci. 15 (2007), $77-81$.

[14] V. Johri and K. Desikan, Cosmological models with constant deceleration parameter in Brans-Dicke theory, Gen. Relativ. Gravitation, 26 (1994), 1217-1232.

[15] P. Jordan, Schwerkraft und Weltall 107, Vieweg, 1955.

[16] R. S. Kumar, Some exact cosmological models in modified theories of gravitation, http://hdl.handle.net/10603/124115, (2013).

[17] L. D. Landau, The classical theory of fields 2, Elsevier, 2013.

[18] G. Magnano, Are there metric theories of gravity other than general relativity, General Relativ. Gravitat. Phys. (1995), 213-1 to 213-19.

[19] S. Nojiri and S. D. Odintsov, Modified gravity with negative and positive powers of curvature: Unification of inflation and cosmic acceleration, Phys. Rev. D, 68 (2003), 123512-1 to 123512-23.

[20] G. Nordstrlom, Trlage und schwere masse in der relativitlats-mechanik, Annalen der Physik, 345 (1913), 856-878.

[21] K. Nordtvedt Jr, Post-newtonian metric for a general class of scalar-tensor gravitational theories and observational consequences., Astrophys. J. 161 (1970), 1059-1068.

[22] P. Sahoo, P. Sahoo, B. K. Bishi, and S. Aygĺun, Magnetized strange quark model with big rip singularity in $f(R, T)$ gravity, Modern Phys. Lett. A, 32 (2017), 1750105-1 to 1750105-19.

[23] D. Sen and K. Dunn, A scalar-tensor theory of gravitation in a modified riemannian manifold, J. Math. Phys. 12 (1971), $578-586$.

[24] J. Wainwright and G. F. R. Ellis, Dynamical systems in cosmology, Cambridge University Press, 2005.

[25] R. M. Wald, General relativity, Chicago, Usa: Univ, Pr. 491, (1984). 